Classification

Physics Abstracts

$07.20-29.20-85.25$

\title{
Enjeux technologiques du LHC, le grand collisionneur de particules supraconducteur en projet au CERN
}

\author{
Ph. Lebrun \\ CERN, Genève, Switzerland \\ (Reçu le 6 mars 1990, accepté le 30 mars 1990)
}

\begin{abstract}
Résumé. - Le projet de collisionneur de particules supraconducteur LHC est présenté, en mettant l'accent sur ses enjeux technologiques majeurs, principalement dans le domaine des électro-aimants à champs intenses, de la cryogénie à l'hélium superfluide et de l'intégration de ces techniques de pointe dans une grande machine. Les actions de coopération et les mécanismes de transfert technologique, en cours ou à créer, vers les laboratoires et les industries européens sont brièvement évoqués.
\end{abstract}

\begin{abstract}
The LHC large superconducting particle collider project is presented, with particular emphasis on its major technological requirements and returns, mostly in the domains of high-field electromagnets, superfluid helium cryogenics, and integration of such advanced techniques in a large machine. The corresponding cooperation and technological transfer to European laboratories and industries and briefly discussed.
\end{abstract}

\section{Introduction.}

Le CERN, Laboratoire Européen pour la Physique des Particules Elémentaires, vient de mettre en service avec succès le grand collisionneur d'électrons et de positons LEP [1]. Cet instrument de physique, actuellement le plus grand du monde, est installé près de Genève dans un tunnel annullaire souterrain d'une circonférence de $26,7 \mathrm{~km}$. Grâce à une énergie de faisceau qui sera graduellement portée jusqu'à $100 \mathrm{GeV}$, le LEP permet de sonder la structure de la matière à l'échelle de $10^{-17} \mathrm{~m}$. Pour explorer la physique à des énergies de l'ordre du TeV (1000 GeV) et étudier les interactions fondamentales entre particules dans des conditions nouvelles, le CERN envisage d'installer ultérieurement dans le tunnel du LEP, au-dessus de la machine existante, un grand collisionneur de protons appelé LHC (Large Hadron Collider) [2,3], dont le système magnétique supraconducteur permettrait de produire les champs magnétiques intenses nécessaires au guidage et à la focalisation des faisceaux très rigides de protons de haute énergie (Fig. 1). Le LHC utiliserait, pour l'essentiel, le génie civil et l'infrastructure technique existants, ainsi que l'ensemble de sources et d'injecteurs du CERN, et ferait ainsi l'économie des postes budgétaires correspondants, qui représentent une fraction importante du coût des grands accélérateurs. En outre, la disponibilité d'un complexe d'injecteurs performant, bien rodé et polyvalent permettrait de réaliser également dans le LHC des collisions d'ions lourds, tandis que la configuration jumelée LEP-LHC donnerait 


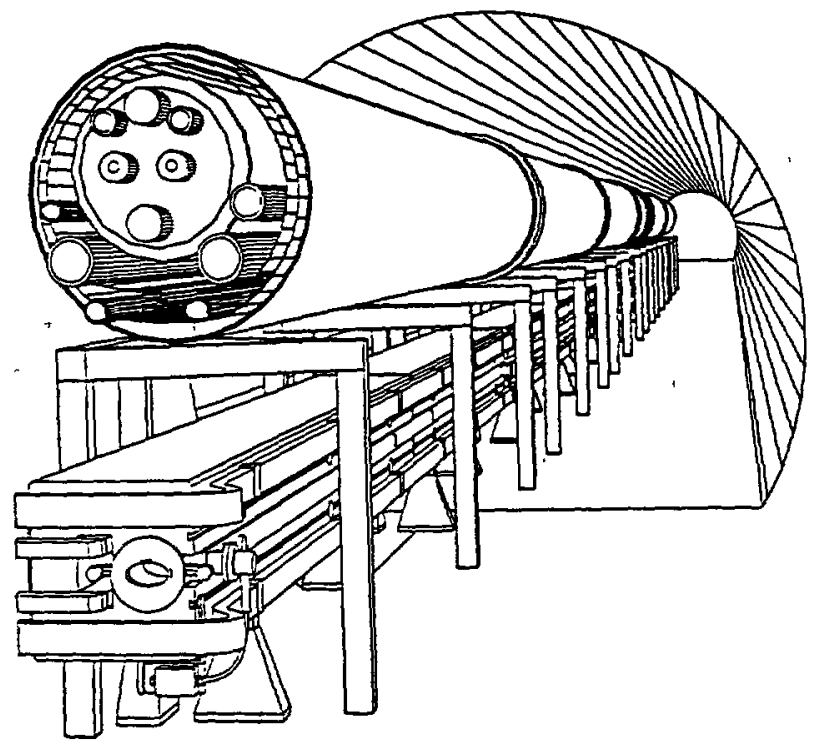

Fig. 1. - Vue du LHC -installé dans le tunnel du LEP.

[View of LHC in the LEP tunnel.]

accès à la physique des collisions électron-proton à haute énergie. En contrepartie de ces avantages, l"obtention d'énergies de faisceau de l'ordre de $8 \mathrm{TeV}$ dans le tunnel existant impose aux aimants supraconducteurs du LHC de produire des champs intenses (environ $10 \mathrm{~T}$ ), à la limite du savoir-faire actuel [4] ; les développements nécessaires dans ce domaine, ainsi que dans les techniques connexes, constituent les enjeux technologiques majeurs du projet LHC.

\section{Configuration générale du LHC.}

Le tunnel du LEP se compose de 8 arcs, séparés par des sections droites longues d'environ $600 \mathrm{~m}$, au milieu desquelles se trouvent les 8 points de collision possibles; les puits d'accès aux ouvrages souterrains et les bâtiments de service, en surface, sont situés au droit de ces 8 points, espacés de $3330 \mathrm{~m}$; le LHC doit respecter cette configuration géométrique de base (Fig. 2). Les 4 points de collision non utilisés pour la physique électron-positon du LEP peuvent ainsi être affectés à de nouvelles zones d'expérimentation proton-proton, ainsi qu'à la décharge des faisceaux du LHC. Les deux faisceaux de protons, circulant en sens inverse et séparés horizontalement de $180 \mathrm{~mm}$ dans les arcs, se croisent et peuvent donc entrer en collision au milieu de chacune des 8 sections droites. Pour des raisons d'encombrement et d'économie, les champs magnétiques de guidage et de focalisation des faisceaux en circulation sont produits par des aimants à ouvertures jumelées, constitués de deux ensembles de bobinages supraconducteurs produisant des champs égaux et opposés et partageant une structure de support mécanique et un circuit magnétique communs. Ces aimants sont inclus dans des cryostats qui abritent également les circuits électriques d'excitation et les canalisations cryogéniques, ce qui minimise l'emprise totale et facilite l'installation dans le tunnel. Le tableau I indique les principaux paramètres du LHC. 


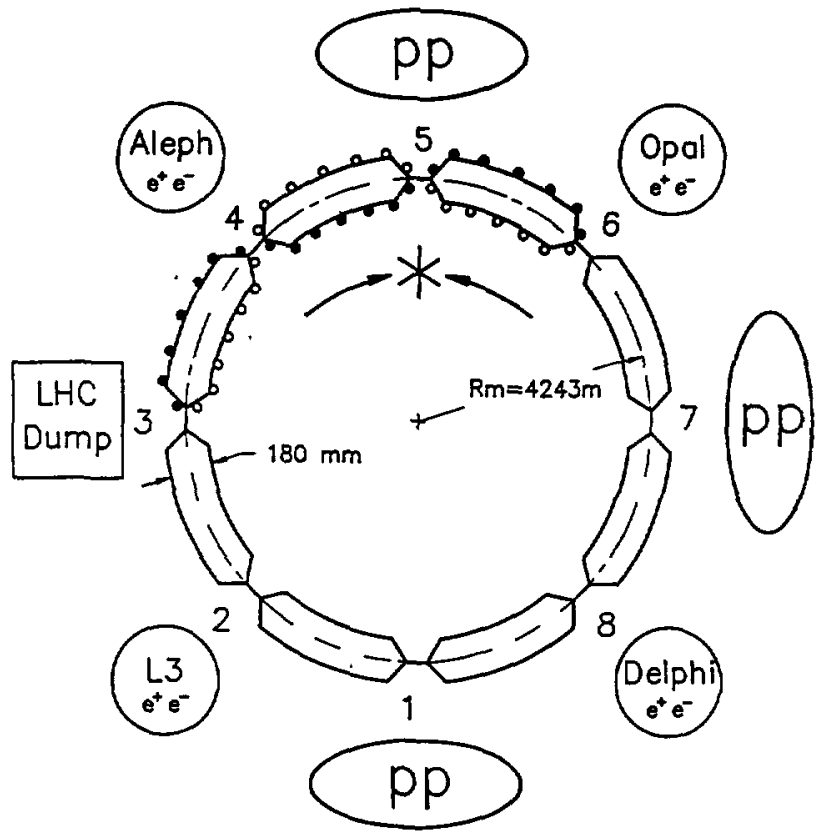

Fig. 2. - Configuration générale de principe du LHC.

[General layout of LHC.]

Tableau I. - Paramètres principaux du LHC, option proton-proton.

[Main parameters of LHC, proton-proton option.]

\begin{tabular}{|l|c|c|}
\hline Circonférence & 26,7 & $\mathrm{~km}$ \\
Champ de courbure nominal & 10 & $\mathrm{~T}$ \\
Energie nominale de faisceau & 8 & $\mathrm{TeV}$ \\
Energie d'injection & 0,45 & $\mathrm{TeV}$ \\
Nombre de paquets de protons & 4810 & \\
Longueur totale d'un paquet & 0,31 & $\mathrm{~m}$ \\
Espacement des paquets & 15 & $\mathrm{~ns}$ \\
Energie 'stockée & $2 \times 597$ & $\mathrm{MJ}$ \\
Puissance synchrotron totale & 18,3 & $\mathrm{MW}$ \\
Luminosité nominale & $3,8 \times 10^{34}$ & $\mathrm{~cm}^{-2} \mathrm{~s}^{-1}$ \\
Temps d'accélération & 1200 & $\mathrm{~s}$ \\
\hline
\end{tabular}

Les arcs, qui représentent la majeure partie du périmètre de la machine, sont constitués de la répétition périodique d'une maille élémentaire (Fig. 3), qui comprend des aimants de courbure (dipôles), de focalisation (quadripôles, sextupôles) et de correction d'orbite, soit en tout près de 5000 composants supraconducteurs dont les caractéristiques principales sont données dans le tableau II ; les quantités correspondantes de matériaux à mettre en œuvre apparaissent dans le tableau III, ce qui permet d'évaluer l'impact industriel important du projet. 

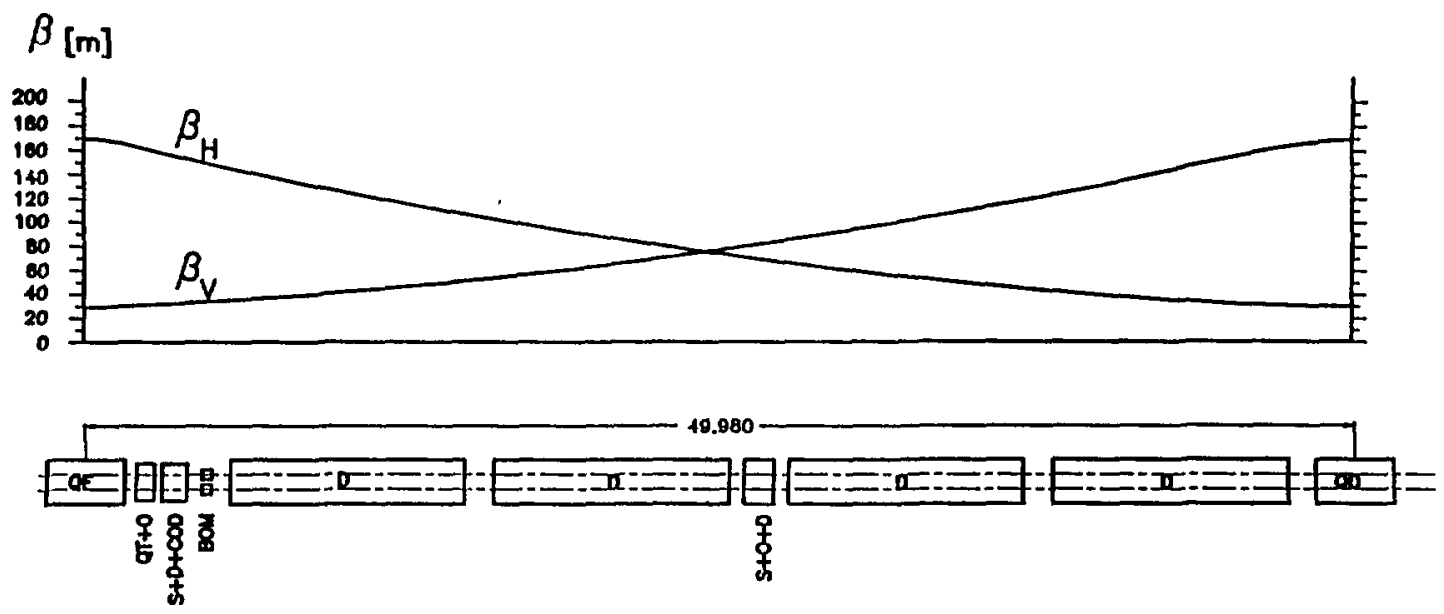

Fig. 3. - Schéma de la maille standard (demi-cellule) du LHC.

[Proposed layout of LHC standard half-cell.]

Tableau II. - Principaux aimants supraconducteurs du LHC.

[Main superconducting magnets in LHC.]

\begin{tabular}{|l|l|r|c|}
\hline Nombre & \multicolumn{1}{|c|}{ Type } & Force & $\begin{array}{c}\text { Longueur } \\
\text { magn. }\end{array}$ \\
\hline $2 \times 1760$ & dipôles & $10 \mathrm{~T}$ & $9,54 \mathrm{~m}$ \\
$2 \times 568$ & quadripôles & $250 \mathrm{~T} \mathrm{~m} \mathrm{~m}^{-1}$ & $3,08 \mathrm{~m}$ \\
796 & quad. d'accord & $120 \mathrm{~T} \mathrm{~m}^{-1}$ & $0,72 \mathrm{~m}$ \\
796 & sextupôles & $3640 \mathrm{~T} \mathrm{~m}^{-2}$ & $1,10 \mathrm{~m}$ \\
398 & correcteurs horiz. & $1,36 \mathrm{~T}$ & $1,10 \mathrm{~m}$ \\
398 & correcteurs vert. & $1,36 \mathrm{~T}$ & $1,10 \mathrm{~m}$ \\
\hline
\end{tabular}

Les composants de la machine équipant les sections droites longues, tels que les quadripôles de focalisation des faisceaux au voisinage des points de collision, sont en nombre plus limité ; ils n'en constituent pas moins, pour certains d'entre eux, des défis technologiques ardus, de part leurs exigences constructives (précision du champ) et leurs contraintes opératoires (niveau de rayonnement).

\section{Technologies clés.}

AIMANTS. - Deux techniques différentes permettent d'atteindre des champs magnétiques de l'ordre de $10 \mathrm{~T}$ : l'utilisation de l'alliage supraconducteur courant $\mathrm{Nb}-\mathrm{Ti}$ à une température inférieure à $2 \mathrm{~K}$ dans l'hélium superfluide, ou celle du composé intermétallique $\mathrm{Nb} 3 \mathrm{Sn}$ au point d'ébullition normal de l'hélium (Fig. 4).

La première méthode repose sur l'emploi d'un conducteur à la technologie éprouvée, qu'il s'agisse de production de fil, de câblage ou de bobinage, mais requiert une cryogénie plus complexe et plus puissante. Outre le fonctionnement à plus basse température, l'utilisation 
Tableau III. - Quantités approximatives de matériaux pour le $L H C$.

[Approximate quantities of materials for LHC.]

\begin{tabular}{|l|c|}
\hline \multicolumn{1}{|c|}{ Matériaux } & $\begin{array}{c}\text { Quantités } \\
\text { (tonnes) }\end{array}$ \\
\hline Supraconducteur (alliage \& cuivre) & 400 \\
Isolants électriques & 180 \\
Espaceurs de cuivre & 260 \\
Colliers d'alliage d'aluminium & 3000 \\
Tôles d'acier à bas carbone & 22000 \\
Autres composants en acier & 3700 \\
Aciers inoxydables austénitiques & 4500 \\
Ecrans thermiques (aluminium) & 1000 \\
\hline Masse totale & 36000 \\
Masse froide (4,5 K ou 1,8 K) & 30000 \\
\hline
\end{tabular}

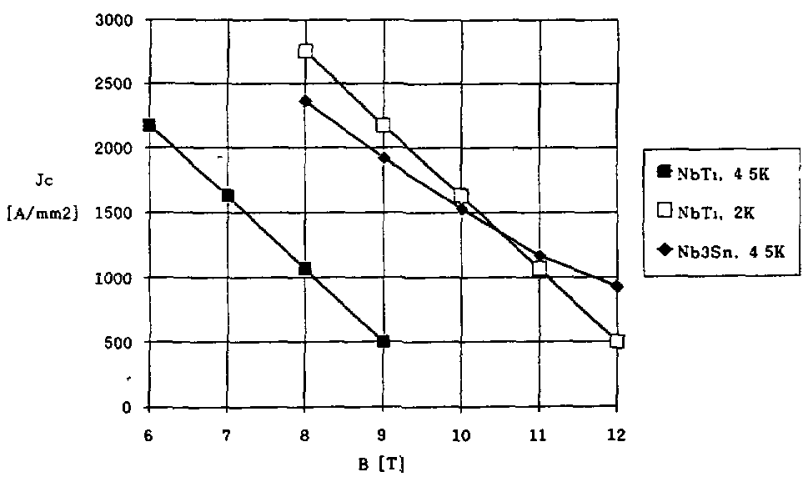

Fig. 4. - Densités de courant critique des supraconducteurs techniques.

[Critical current densities of technical superconductors.]

d'hélium superfluide, de faible viscosité, imprégnant un bobinage à la structure poreuse permet, grâce à sa haute conductibilité thermique et sa grande chaleur spécifique, une bonne stabilisation du supraconducteur, en dépit d'une marge de température plus faible.

L'avantage principal de la seconde méthode réside dans la mise en œuvre d'une cryogénie classique, plus simple, au prix d'une complication accrue dans les techniques de bobinage, d'isolation électrique et de traitement thermique des bobines : la grande fragilité du composé Nb3Sn impose en effet de le produire par diffusion au cceur du bobinage déjà réalisé, au moyen d'un recuit de longue durée à des températures d'environ $700{ }^{\circ} \mathrm{C}$ (technique "windand-react "). La marge de température plus élevée du supraconducteur $\mathrm{Nb3Sn}$, ainsi que l'augmentation rapide des chaleurs spécifiques avec la température, confèrent néanmoins à 
cette solution un intérêt particulier pour les régions du LHC soumises à des dépôts d'énergie instantanés créés par les pertes de faisceau.

Le long de ces deux lignes d'approche, des résultats prometteurs ont déjà été obtenus [5] sur des modèles courts $(1 \mathrm{~m})$ de dipôles supraconducteurs à ouverture unique $[6,7,8]$. Des modèles courts [9] et un prototype à pleine échelle [10] de dipôles à ouvertures jumelées sont en cours de réalisation dans l'industrie, et doivent être testés en 1990.

Néanmoins, des développements complémentaires sont nécessaires pour atteindre des densités de courant critique supérieures, pour produire économiquement des conducteurs à filaments fins (de l'ordre de $5 \mu \mathrm{m}$ ) afin de minimiser les effets des courants rémanents, pour réaliser industriellement des câbles plats de section trapézoïdale à la géométrie bien contrôlée, sans dégradation de performance du supraconducteur, pour mettre au point des isolants électriques préservant la perméation de l'hélium superfluide tout en résistant aux sollicitations mécaniques énormes auxquelles ils sont soumis lors de l'assemblage, de la mise en froid et de l'excitation des enroulements supraconducteurs.

Outre les développements techniques liés aux aimants eux-mêmes; la réalisation d'une machine complète pose les problèmes de leur raccordement en série dans le tunnel, de leur alimentation électrique et de leur protection en cas de transition résistive accidentelle ("quench»), questions rendues d'autant plus ardues par la forte valeur du courant d'excitation (15 kA) ; en particulier, la dérivation du courant principal au niveau d'un aimant de la chaîne ayant subi une transition résistive implique le développement de dispositifs de commutation rapide (diodes) capables de courants forts, fonctionnant à basse température et résistant aux rayonnements ionisants.

Enfin, le contrôle de qualité d'unẹ production en série de milliers de composants complexes de provenances diverses exige la mise en cuvre de techniques spécifiques, en particulier pour réaliser des mesures magnétiques (courbe d'excitation, composantes multipolaires, champ rémanent) au cour des ouvertures utiles des aimants supraconducteurs.

CryogénIE. - Les travaux ont porté principalement sur l'étude du système cryogénique à hélium superfluide [11], dont la faisabilité conditionne de manière rédhibitoire l'option $\mathrm{Nb}-\mathrm{Ti}$ du projet. La technologie de l'hélium superfluide pressurisé, et sa mise en cuvre pour refroidir des dispositifs supraconducteurs, ont été inventées et développées au CEN Grenoble $[12,13]$, avant de trouver leur application à grande échelle dans des projets comme le tokamak Tore Supra [14] au CEN Cadarache.

Les fonctions essentielles du système cryogénique du LHC consistent à maintenir tous les aimants à une température inférieure à $1,95 \mathrm{~K}$ en fonctionnement stationnaire ou pulsé, à refroidir l'ensemble de la machine depuis la température ambiante en moins de 20 jours, et à remettre en froid une chaîne d'aimants courte $(50$ à $100 \mathrm{~m})$ en quelques heures après une transition résistive accidentelle.

Le système cryogénique doit également satisfaire aux contraintes générales du LHC, à savoir l'implantation dans le génie civil existant, la pente du tunnel $(1,4 \%$ par rapport à l'horizontale), la distance entre les points d'accès, qui implique la distribution de puissance de réfrigération sur $1660 \mathrm{~m}$, la section limitée et l'encombrement du tunnel, et la minimisation des interférences géométriques et opératoires avec le LEP.

Les charges thermiques auxquelles est soumis le système cryogénique en régime permanent trouvent leur origine dans trois types de processus (Tab. IV) :

- les entrées de chaleur, qui dépendent principalement de la conception des cryostats; la figure 5 montre, en coupe transversale, un exemple de cryostat de dipôle basé sur trois niveaux de température nominaux, soit $80 \mathrm{~K}$ (écran externe et premier intercept thermique des supports d'aimant), $5 \mathrm{~K}$ (écrans internes et second intercept thermique des supports) et $1,8 \mathrm{~K}$ (enceinte à hélium et aimant supraconducteur), 
Tableau IV. - Estimation des charges thermiques distribuées en régime permanent dans les dipôles principaux $\left(\mathrm{W} \cdot \mathrm{m}^{-1}\right)$.

[Estimation of distributed steady-state heat loads in the main dipoles (W.m $\left.{ }^{-1}\right)$.]

\begin{tabular}{|l|c|c|c|}
\hline \multicolumn{1}{|c|}{ Température } & $80 \mathrm{~K}$ & $5 \mathrm{~K}$ & $1,8 \mathrm{~K}$ \\
\hline Entrées de chaleur $\left(^{*}\right)$ & 6 & 0,4 & 0,15 \\
Dissipation résistive & - & - & 0,15 \\
Pertes dues au faisceau & - & 0,9 & 0,01 \\
\hline Total (**) & 6 & 1,3 & 0,31 \\
\hline
\end{tabular}

$\left.{ }^{*}\right)$ sans facteur de sécurité.

$\left.{ }^{* *}\right)$ non comprises les charges thermiques localisées.

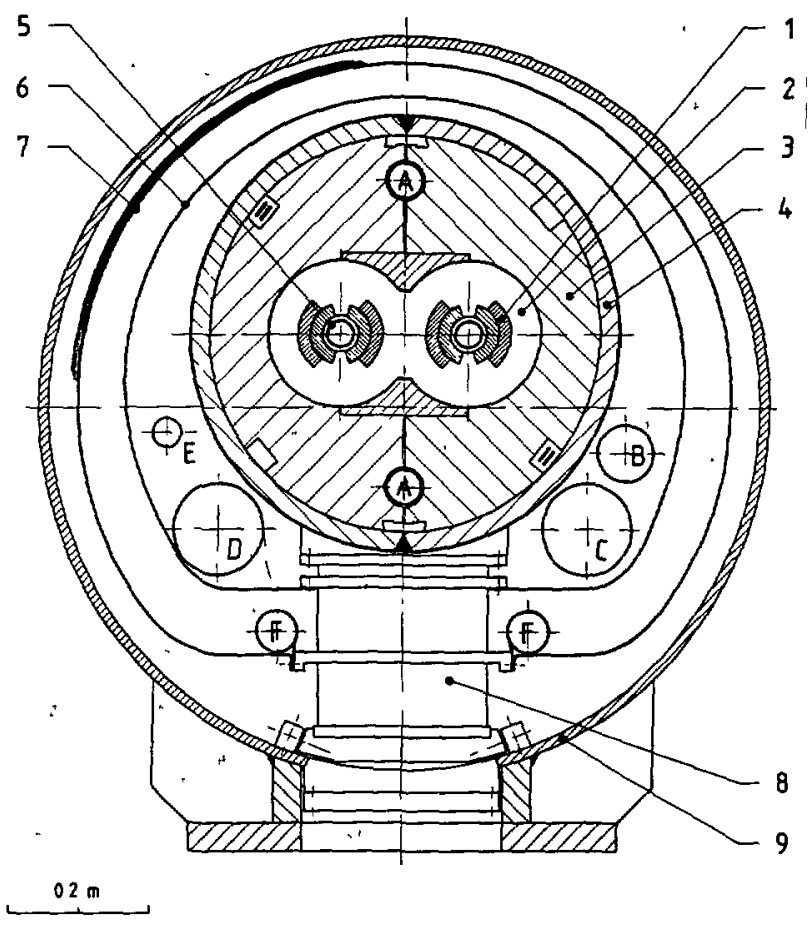

Fig. 5. - Coupe type d'un dipôle LHC dans son cryostat : (1) bobines supraconductrices ; (2) colliers de serrage ; (3) circuit magnétique ; (4) cylindre de frettage ; (5) tube de faisceau ; (6) écran $5 \mathrm{~K}$; (7) écran $80 \mathrm{~K} ;(8)$ pied-colonne; (9) enceinte à vide.

[Standard cross-section of LHC 'dipole in its cryostat: (1) superconducting coils ; (2) collars; (3) magnetic circuit ; (4) shrinking cylinder; (5) beam pipe; (6) $5 \mathrm{~K}$ shield ; (7) $80 \mathrm{~K}$ shield ; (8) support post ; (9) vacuum vessel.] 
- la dissipation résistive dans les capteurs d'instrumentation et les jonctions nonsupraconductrices,

- les dépositions d'énergie liées à la circulation des faisceaux de protons (rayonnement synchrotronique, courants induits, pertes de particules), qui sont, pour la plupart d'entre elles, interceptées par les écrans internes à $5 \mathrm{~K}$.

Les charges thermiques additionnelles qui apparaissent en régime transitoire (montée et descente du courant principal) bien qu'importantes, sont absorbées par la capacité calorifique de l'hélium liquide contenu dans les cryostats.

A partir des estimations précédentes, il est possible d'évaluer les puissances de réfrigération à installer dans chacun des 8 réfrigérateurs d'octant du LHC, soit environ $1,75 \mathrm{~kW}$ à $1,8 \mathrm{~K}$, $7 \mathrm{~kW}$ plus $9 \mathrm{~g} / \mathrm{s}$ (non-isotherme) à $4,5 \mathrm{~K}$, et $30 \mathrm{~kW}$ à une température inférieure à $80 \mathrm{~K}$; ces machines, d'une puissance équivalente de l'ordre de $15 \mathrm{~kW}$ à $4,5 \mathrm{~K}$, compteraient parmi les plus grosses installations cryogéniques existantes.

La courbe de saturation de l'hélium impose d'abaisser la pression à des valeurs de l'ordre du $\mathrm{kPa}$ ( 10 mbar) pour atteindre des températures inférieures à $2 \mathrm{~K}$; les cycles thermodynamiques des réfrigérateurs d'octant devront donc utiliser des compresseurs froids [15], technologie nouvelle mise au point par l'industrie à l'occasion des installations cryogéniques à hélium superfluide des projets Tore Supra [14] et CEBAF [16].

Pour distribuer la puissance frigorifique dans le tunnel le long des demi-octants adjacents à chaque réfrigérateur, le schéma de la figure 6 a été envisagé : une pompe froide fait circuler un débit d'hélium superfluide pressurisé le. long de la chaîne d'aimants, dont les charges thermiques sont absorbées par la capacité calorifique du fluide, au prix d'une élévation de température; pour limiter celle-ci, le débit d'hélium en circulation est périodiquement refroidi par échange thermique avec des bains d'hélium superfluide saturé localisés dans des

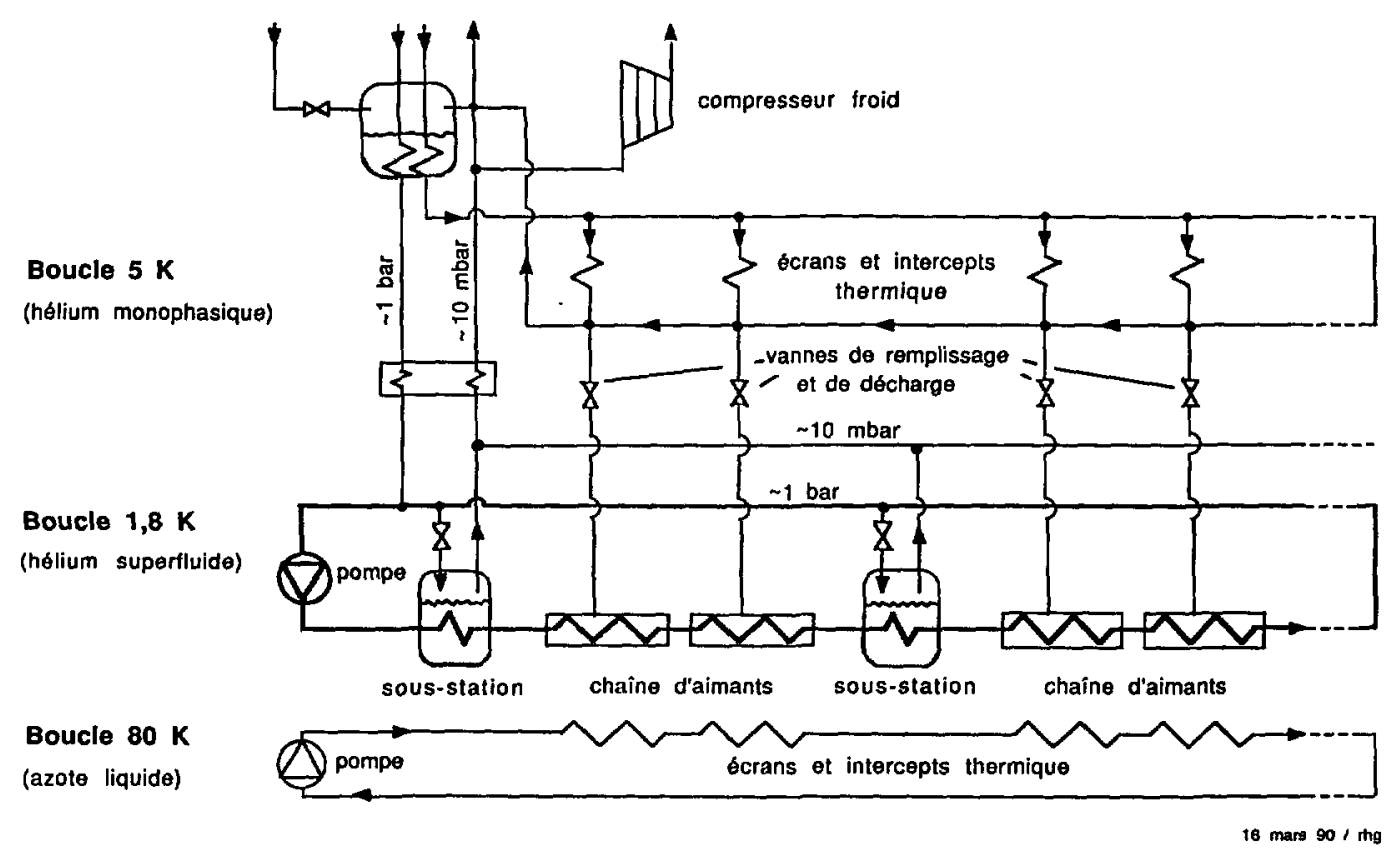

Fig. 6. - Schéma de distribution cryogénique à circulation forcée d'hélium superfluide pressurisé. [Cryogenic distribution scheme based on forced circulation of pressurised superfluid helium.] 
sous-stations de réfrigération installées dans le tunnel, et pompés depuis les réfrigérateurs d'octant à travers une canalisation froide de grand diamètre $(150 \mathrm{~mm})$, incluse dans les cryostats d'aimants. Une alternative potentiellement très intéressante, qui permettrait de s'affranchir des circuits en dépression et du système de pompage, est constituée par l'utilisation de réfrigérateurs magnétiques [17] extrayant quelques dizaines de watts entre 1,8 et $4,5 \mathrm{~K}$, et équipant les sous-stations du tunnel. La démonstration de principe en a été faite sur des machines de laboratoire, d'une puissance de l'ordre du watt; un développement, utilisant les progrès récents dans la mise au point de supraconducteurs à faibles pertes en régime variable, est en cours pour réaliser un prototype adapté aux exigences du LHC.

Du fait de la masse froide considérable et des tolérances serrées d'alignement du LHC, un des facteurs critiques de la performance du cryostat se trouve être le système de supportage de laimant, qui doit faire appel à la technologie des matériaux composites pour pouvoir concilier ses exigences géométriques, mécaniques et thermiques; des prototypes de supports en composite fibre de verre-époxy, réalisés dans l'industrie, ont été qualifiés, et le développement de composants en matériaux, plus perfectionnés est en cours.

Enfin, la faisabilité technologique d'un système cryogénique réparti sur près de $27 \mathrm{~km}$ et comportant plus de 40000 soufflets et raccords repose sur la maitrise des techniques de construction d'enceintes à pression, de garantie d'étanchéité (soudage automatique in situ) et de contrôle de qualité (qualification des fournisseurs, réception des composants, procédures d'assurance qualité, essais non-destructifs, détection de fuites in situ), ainsi que sur la mise au point de méthodes d'installation spécifiques.

\section{Coopération et transfert de technologie.}

Il a toujours été dans la tradition du CERN de s'appuyer sur la compétence des laboratoires nationaux et le savoir-faire des industries europêennes pour mener à bien ses grands projets

Tableau V. - Laboratoires européens participant aux activités de recherche et développement pour le' $L H C$.

[European laboratories involved in research and development activities for LHC.]

\begin{tabular}{|l|l|}
\hline Autriche & Univ. Tech. de Graz \\
Belgique & Univ. Tech. de Vienne \\
France & CEN Mol \\
& CEA Cadarache \\
& CEA Grenoble \\
Allemagne (R.F.A.) & CEA Saclay \\
& DESY Hambourg \\
Italie & KfK Karlsruhe \\
& INFN Frascati \\
INFN Gênes \\
Pays-Bas & INFN Milan \\
& NIKHEF Amsterdam \\
& ECN Petten \\
Suisse & Univ. de Twente \\
Royaume-Uni & FOM Utrecht \\
& PSI Villigen \\
& RAL Didcot \\
\hline
\end{tabular}


d'accélérateurs et de détecteurs. Le projet LHC perpétue et renforce cette tradition, en impliquant ces partenaires dès la phase de recherche et développement, bien en amont de la construction de la machine proprement dite. Cette approche novatrice a rencontré jusqu'ici un écho très favorable, tant auprès des laboratoires que des industries, qui, bénéficiant du récent regain d'intérêt pour la supraconductivité, ont pu acquérir le savoir-faire approprié en participant à de grảnds projets comme le collisionneur HERA de Hambourg ou le tokamak Tore Supra de Cadarache. De fait, dix-sept laboratoires européens (Tab. V) et une vingtaine d'industries (Tab. VI) sont actuellement engagés dans le programme de recherche et développement pour le LHC, dans le cadre d'accords de coopération ou de contrats commerciaux, avec dès premiers résultats très positifs. En procédant de la sorte, le CERN contribue à créer en Europe une base technologique appropriée à la construction industrielle de grands dispositifs supraconducteurs qui, au-delà de la réalisation éventuelle du LHC, renforcera la compétence dans ce domaine et profitera à l'ensemble du secteur industriel concerné.

Tableau VI. - Activités de recherche et développement pour le LHC dans l'industrie européenne.

[Research and development activities for LHC in european industry.]

\begin{tabular}{|l|c|c|}
\hline Conducteurs et câbles Nb-Ti & ABB & Suisse \\
& Alsthom & France \\
& LMI & Italie \\
Conducteurs et câbles Nb3Sn & VAC & Allemagne \\
& Pays-bas \\
& IMI & Royaume-Uni \\
& Plansee & Autriche \\
Dipôles courts Nb-Ti, ouverture unique & VAC & Allemagne \\
Dipôles courts Nb-Ti, ouvertures jumelées & Ansaldo & Italie \\
& Jeumont & France \\
& ELIN & Autriche \\
& Holec & Pays-Bas \\
Dipôle prototype long Nb-Ti & Ansaldo & Italie \\
Dipôle court Nb3Sn, ouverture unique & ABB & Allemagne \\
Dipôle court Nb3Sn, ouvertures jumelées & ELIN & Autriche \\
Quadripôles & Holec & Pays-Bas \\
Sextupôles et dipôles de correction & CEA & France \\
Quadripôles d'accord & Tesla' & Royaume-Uni \\
Cryostat long à hélium superfluide & ACICA & Espagne \\
Diodes froides à courant fort & FBM & Italie \\
& ABB & Suisse \\
Isolants électriques & Marconi & Royaume-Uni \\
& Isola & Suisse \\
\hline
\end{tabular}

\section{Conclusion.}

La conception, la construction et l'exploitation de grands instruments de physique, points de passage obligés de la recherche fondamentale, n'en constituent pas moins des aventures 
industrielles passionnantes: les défis technologiques qu'elles soulèvent se révèlent des moteurs de développement dynamiques, tandis que l'importance des budgets mis en jeu représente des marchés intéressants à l'échelle de l'industrie européenne. Le projet LHC, estimé à environ 7 milliards de francs français dont $80 \%$ pour la cryogénie et la supraconductivité, en est un exemple caractéristique.

\section{Bibliographie}

[1] Bächy G., Hofmann A, Myers S., Picasso E. and Plass G., The LeP collider : construction, project status and outlook, Proc. XIV Int. Conf. on High-Energy Accelerators (Tsukuba) 1989.

[2] BRIANTI G. and HUUBNER K., Eds., The Large Hadron Collider in the LEP tunnel, CERN (1987) 87-05.

[3] Brianti G., The Large Hadron Collider (LHC) in the LEP tunnel, Proc. XIV Int. Conf. on HighEnergy Accelerators (Tsukuba) 1989.

[4] PERIN R., State of the art in high-field superconducting magnets for particle accelerators, Proc. XIV Int. Conf. on High-Energy Accelerators (Tsukuba) 1989.

[5] PERIN R., First results of the high-field magnet development for the Large Hadron Collider, Proc. 11 th Int. Conf. on Magnet Technology (Tsukuba) 1989.

[6] Perin R., Leroy D. and SPIGo G., The first industry-made model magnet for the CERN LHC, IEEE Trans. Magnetics 25 (1989) 1632-1635.

[7] ASNer A., WENGer S. and Zerobin F., Towards a $1 \mathrm{~m}$-long high-field Nb3Sn dipôle magnet of the CERN-ELIN collaboration, IEEE Trans. Magnetics, 25 (1989) 1636-1639.

[8] Asner A., Perin R., Wenger S. and Zerobin F., First Nb3Sn, 1 m-long superconducting dipôle magnet for LHC break the 10 tesla field threshold, Proc. 11th Int. Conf. on Magnet Technology (Tsukuba) 1989.

[9] Leroy D., Perin R., DE RIJK G. and Thomi W., Design of a high-field twin-aperture superconducting dipôle model, IEEE Trans. Magnetics 24 (1988) 1373-1376.

[10] Hagedorn D., Lebrun Ph., Leroy D., Perin R., Vlogaert J. and McInturfF A., Design and construction of a twin-aperture prototype magnet for the CERN LHC project, Proc. 11th Int. Conf. on Magnet Technology (Tsukuba) 1989.

[11] Claudet G., Disdier F., Gauthier A., Lebrun Ph., Morpurgo M. and Schmid J., Conceptual study of the superfluid helium cryogenic system for the CERN Large Hadron Collider (LHC), Proc. ICEC12, Butterworth (1988) 497-504.

[12] Bon Mardion G., Claudet G., Seyfert P. and Verdier J., Helium II in low-temperature and superconductive magnet engineering, Adv. Cryo. Eng. 23 (1978) 358-362.

[13] Claudet G. and Aymar R., Tore Supra and the helium II cooling of large high-field magnets, Proc. CEC89 (Los Angeles) 1989.

[14] Claudet G., Aymar R., Bon Mardion G., Jager B. and Gistau G., Le système cryogénique de Tore Supra, Comptes Rendus des Deuxièmes Journées d'Aussois «Cryogénie et Supraconductivité » (1986).

[15] Gistau G., Villard J.C. and TuRCat F., Range of application of cryogenic centrifugal compressors, Proc. CEC89 (Los Angeles) 1989.

[16] Rode C. H., 2.0 K CEBAF Cryogenics, Proc. CEC89 (Los Angeles) 1989.

[17] SeYfert P., Research on magnetic refrigeration at CEA Grenoble, Proc. CEC89 (Los Angeles) 1989. 